\title{
Magnetic invisibility of the magnetically coated type-II superconductor in partially penetrated state
}

\author{
J. Peña-Roche, ${ }^{1}$ Y. A. Genenko, ${ }^{2}$ and A. Badía-Majós ${ }^{1, a)}$ \\ ${ }^{1}$ Departamento de Física de la Materia Condensada and Instituto de Ciencia de Materiales de Aragón \\ (ICMA), Universidad de Zaragoza-CSIC, C/María de Luna 3, E-50018 Zaragoza, Spain \\ ${ }^{2}$ Institut für Materialwissenschaft, Technische Universität Darmstadt, Jovanka-Bontschits Str. 2, \\ D-64287 Darmstadt, Germany
}

(Received 19 July 2016; accepted 14 August 2016; published online 29 August 2016)

\begin{abstract}
The magnetic behavior of a cylindrical paramagnet/superconductor heterostructure has been studied by numerical simulations. Relying on the variational statement of the critical state model, our results show that magnetic invisibility is compatible with the partial penetration regime in the superconductor. This result accomplishes previous analytic studies that proved a possible perfect undetectability for the full penetration of magnetic flux. For a given geometry, invisibility may be realized only at a certain magnitude of the applied field. Such value decreases with increasing permeability of the magnetic sheath and eventually collapses to zero. This establishes a condition for obtaining realizable invisibility that extends previous expectations relying either on the full penetration ansatz or on perfect diamagnetism. Published by AIP Publishing.
\end{abstract}

[http://dx.doi.org/10.1063/1.4961672]

Interplay of physical properties of superconductors (SCs) and ferromagnets (FMs) in smart SC/FM heterostructures provides a range of unusual properties of these metamaterials. ${ }^{1}$ At the macroscopic level, combining the diamagnetic properties of SCs and magnetization of paramagnets in bilayer and multilayer structures allows for such phenomena as magnetic cloaking of static ${ }^{2,3}$ and low frequency ${ }^{4,5}$ magnetic fields. Over and above, similar structures provide transportation of magnetic flux with low leakage (magnetic hose $)^{6,7}$ and great reduction of ac losses when exposed to an external ac field. ${ }^{8}$ While demonstrating a theoretical plausibility of such devices supported by a proof-of-principle experiments, ${ }^{3-7}$ the model description of the superconducting component still remains strongly simplified, using typically the approximation of the ideal diamagnet with the permeability $\mu=0$. This prevents completely the penetration of magnetic flux into the superconducting constituents and strongly affects the expected device performance. Account of magnetic field penetration within the London theory ${ }^{9}$ and in the full critical state ${ }^{10}$ covers, respectively, the limiting cases of very weak and very strong magnetic fields leaving open a wide intermediate range of fields in which the superconductor is partially penetrated by magnetic flux. This much more complicate task can only be resolved by a numerical treatment which presents a focus of the current work.

We consider the response of a long superconducting wire (circular cross section), covered by a concentric layer of paramagnetic material. The system is exposed to a uniform external magnetic field $\mathscr{H}_{0}$ perpendicular to its axis (z-axis in what follows). This structure is simpler than cloaking arrangements studied in Refs. 2-5; however, it allows a vivid demonstration of the impact of partial penetration of magnetic flux on cloaking conditions.

\footnotetext{
${ }^{\text {a) }}$ Author to whom correspondence should be addressed. Electronic mail: anabadia@unizar.es
}

The behavior of the heterostructure is dealt with as follows. The superconductor is a hard type-II material, modeled through the critical state ansatz, i.e., by means of the critical current density parameter $J_{c}$ as introduced by Bean. ${ }^{11}$ Since in the considered geometry, a simple quasi-one-dimensional treatment is not possible, a numerical procedure is used to resolve the quasistationary response to the external excitation. As for the magnetic material, which is assumed to be a linear medium, continuity of the tangential component of the magnetic field intensity $\overrightarrow{\mathscr{H}}=\overrightarrow{\mathscr{B}} / \mu$ is invoked at the interfaces. Both material properties are introduced in a selfconsistent algorithm.

Attention has been paid to the so-called full penetration and cloaking fields of the system. They are defined, respectively, by the values for which the superconducting magnetic moment either saturates $\left(\mathscr{H}_{0}=\mathscr{H}_{\mathrm{fp}} \Rightarrow m_{\mathrm{sc}}=m_{\mathrm{sc}}^{\max }\right)$ or exactly cancels the response of the magnet $\left(\mathscr{H}_{0}=\mathscr{H}_{\text {cloak }}\right.$ $\Rightarrow m_{\mathrm{sc}}+m_{\mathrm{mag}}=0$ ). In the case considered here (applied uniform field), when the responses of the superconductor and the magnet are both dipolar, this condition means full magnetic invisibility because all higher magnetic moments equal zero.

The configuration of the composite is described in terms of current density functions $J_{\mathrm{sc}}(\vec{r}, t)$ and $J_{\text {mag }}(\vec{r}, t)$. They correspond, respectively, to the superconductor's macroscopic current density and to the conventional effective magnetization current density for the case of the magnet. By symmetry, both vectors are directed along the axis of the wires and constant along such direction, i.e., $\vec{J}=\left(0,0, J_{z}(x, y)\right)$.

From the technical point of view, a finite element formulation has been considered. We start with a suitable discretization of the circular multiple domain of the problem, i.e., $\left\{\vec{r}_{i}=\left(x_{i}, y_{i}\right) i=1 \ldots N_{G}\right\}$. This will be the support of the $N_{G^{-}}$ dimensional vector quantities formed by the current density values at the grid points. They will be named after Dirac's "bra" and "ket" notation, with $\langle j|$ the transpose of $|j\rangle$. In 
practice, the superconducting and magnetic sub-domains will be treated separately, i.e., $|j\rangle \equiv\left|j_{\mathrm{sc}}\right\rangle \oplus\left|j_{\mathrm{mag}}\right\rangle$.

Notice that, whereas the physical counterpart of $\left|j_{\mathrm{sc}}\right\rangle$ is a set of filaments that discretize the cross section of the superconductor, $\left|j_{\mathrm{mag}}\right\rangle$ will be better implemented as a polygonal approximation in terms of an array of thin strips along the magnet's boundaries. In fact, owing to the linearity of the magnetic material, effective current densities are described by surface layers at the interfaces. In brief, $\overrightarrow{\mathscr{B}}=\mu \overrightarrow{\mathscr{H}} \Rightarrow$ $\nabla \times \overrightarrow{\mathscr{M}}=0$ and then $J_{\text {mag }}(\vec{r})$ takes the form of surface current densities given by $\overrightarrow{\mathscr{M}} \times \hat{n}^{12}$ at the discontinuities of magnetization, i.e., at the boundaries of the sheath. More specifically, for the case of our magnetic layer, a natural decomposition is $\left|j_{\text {mag }}\right\rangle \equiv\left|j_{\text {in }}\right\rangle \oplus\left|j_{\text {out }}\right\rangle$, standing for the concatenation of surface current density elements at the inner and outer boundaries.

Several strategies for modeling the response of the heterostructure were investigated. A numerically stable algorithm that updates the system, subject to some definite external process $\left(\mathscr{H}_{0}\left(t_{n}\right) \rightarrow \mathscr{H}_{0}\left(t_{n+1}\right)\right)$, was found to be the double step iterative flow chart in (1)

$$
\begin{array}{lll}
\left|j_{\mathrm{sc}}^{(n)}\right\rangle & \text { and } & \left|j_{\text {mag }}^{(n)}\right\rangle \text { are given, then : } \\
\left|j_{\mathrm{sc}}^{(n)}\right\rangle & \stackrel{\text { boundary condition }}{\longrightarrow} & \mid j_{\mathrm{mag}}^{(n+1)} \\
\mathscr{H}_{0}^{(n+1)} & \stackrel{ }{\longrightarrow} & \mid j_{\mathrm{sc}}^{(n+1)} \\
\left|j_{\mathrm{mag}}^{(n+1)}\right\rangle & \stackrel{\text { variational principle }}{\longrightarrow} & \\
\mathscr{H}_{0}^{(n+1)} &
\end{array}
$$

and so on...

Here, $\left|j_{\text {sc }}^{(n)}\right\rangle$ means the current density vector at the grid points of the superconductor, and time step $n$ (analogously for $\left|j_{\text {mag }}^{(n)}\right\rangle$ in the magnet). Each step is resolved as follows.

As shown in the previous work, ${ }^{13}$ the updated configuration of supercurrents $\left|j_{\mathrm{sc}}^{(n+1)}\right\rangle$ may be obtained by minimizing the following expression of the free energy:

$$
\begin{aligned}
\mathrm{F}\left[\left|j_{\mathrm{sc}}^{(n+1)}\right\rangle\right]= & \frac{1}{2}\left\langle j_{\mathrm{sc}}^{(n+1)}|\mathrm{M}| j_{\mathrm{sc}}^{(n+1)}\right\rangle-\left\langle j_{\mathrm{sc}}^{(n)}|\mathrm{M}| j_{\mathrm{sc}}^{(n+1)}\right\rangle \\
& +\left\langle\mathscr{A}_{0}^{(n+1)}-\mathscr{A}_{0}^{(n)} \mid j_{\mathrm{sc}}^{(n+1)}\right\rangle+\left\langle j_{\mathrm{mag}}^{(n+1)}-j_{\mathrm{mag}}^{(n)}|\mathrm{P}| j_{\mathrm{sc}}^{(n+1)}\right\rangle .
\end{aligned}
$$

In this equation, $\mathrm{M}$ represents the $N_{\mathrm{sc}} \times N_{\mathrm{sc}}$ mutual inductance matrix, between the superconducting elements, and $\left\langle\mathscr{A}_{0}^{(n+1)}-\mathscr{A}_{0}^{(n)}\right|$ is the $1 \times N_{\mathrm{sc}}$ row vector, formed by the increment of applied vector potential (z-component) at the grid points of the superconductor $\left(\nabla \times \overrightarrow{\mathscr{A}}_{0}=\mu_{0} \overrightarrow{\mathscr{H}}_{0}\right)$. Finally, P is the $N_{\text {mag }} \times N_{\text {sc }}$ mutual inductance matrix that couples the current density elements within the magnet and the superconductor. We have derived

$$
\begin{gathered}
M_{i j}=\frac{\mu_{0}}{2 \pi} \ln \frac{a^{2}}{x_{i j}^{2}+y_{i j}^{2}}, M_{i i}=\frac{\mu_{0}}{8 \pi}, \\
P_{i j}=\frac{\mu_{0}}{8 \pi}\left\{\left(x_{i j}-L\right) \ln \left[\left(x_{i j}-L\right)^{2}+y_{i j}^{2}\right]+\left(x_{i j}+L\right)\right. \\
\left.\times \ln \left[\left(x_{i j}+L\right)^{2}+y_{i j}^{2}\right]-2 y_{i j}\left[\operatorname{atan} \frac{L-x_{i j}}{y_{i j}}+\operatorname{atan} \frac{L+x_{i j}}{y_{i j}}\right]\right\} .
\end{gathered}
$$

Here, the following notation has been used: $x_{i j} \equiv x_{i}-x_{j}$, $y_{i j} \equiv y_{i}-y_{j}$ give the relative position between elements $i$ and $j ; a$ (gauge for our length units) is the radius of a finite element wire in the superconductor and $L$ the half-width of a finite-element platelet at the magnet's surface. Implicit to our approximation is the fact that the current density is uniform within the elements. Also of mention is that a global constant, related to cross dimension of the infinite wires, occurs to be irrelevant in the variational statement.

As said above, $\left|j_{\text {mag }}\right\rangle$ may be obtained by recalling tangential continuity of the magnetic field vector at the interfaces, i.e.

$$
\mathscr{B}_{t}^{+} / \mu^{+}=\mathscr{B}_{t}^{-} / \mu^{-},
$$

with,+- used to indicate the position of two neighboring points, one on each side of the given boundary. ${ }^{14}$

The above expression may be easily transformed into a linear algebraic system with $\left|j_{\text {mag }}\right\rangle$ the vector of unknowns. Taking advantage of linearity and Biot-Savart's law for the magnetic sources, one gets

$$
\left(\mathrm{G}_{\mathrm{mag}}^{+}-\mu_{r} \mathrm{G}_{\mathrm{mag}}^{-}\right)\left|j_{\text {mag }}^{(n+1)}\right\rangle=\left(\mu_{r}-1\right)\left|\mathscr{H}_{0 m}^{(n+1)}\right\rangle+\left(\mu_{r}-1\right) \mathrm{G}_{\mathrm{sc}}\left|j_{\mathrm{sc}}^{(n)}\right\rangle .
$$

Here, G's stand for the geometry dependent matrices that provide the tangential magnetic field values created by the current densities at the grid points of interest (here, the interfaces). On the other hand, $\mathscr{H}_{0 m}$ stands for the tangential component of applied magnetic field. The actual expressions used to generate the matrix elements $G_{\text {mag }}$ rely on the following formulas for the magnetic field created by a thin strip (of width $L$, spanning along the $z$-axis) that carries a uniform surface current density $j_{\text {surf }}$

$$
\begin{aligned}
& \mathscr{B}_{x}^{\prime}\left(x^{\prime}, y^{\prime}\right)=-\frac{\mu_{0} j_{\text {surf }}}{2 \pi}\left[\operatorname{atan} \frac{x^{\prime}-L / 2}{y^{\prime}}-\operatorname{atan} \frac{x^{\prime}+L / 2}{y^{\prime}}\right] \\
& \mathscr{B}_{y}^{\prime}\left(x^{\prime}, y^{\prime}\right)=\frac{\mu_{0} j_{\text {surf }}}{4 \pi} \ln \frac{\left(x^{\prime}-L / 2\right)^{2}+y^{\prime 2}}{\left(x^{\prime}+L / 2\right)^{2}+y^{\prime 2}} .
\end{aligned}
$$

Here, local coordinates $\left(x^{\prime}, y^{\prime}\right)$ relative to the center of each elementary strip are used.

As regards the superconducting contribution, the matrix $\mathrm{G}_{\mathrm{sc}}$ is evaluated on the basis of the infinite wire approximation, i.e., from

$$
\overrightarrow{\mathscr{B}}_{\mathrm{sc}}\left(x^{\prime}, y^{\prime}\right)=\frac{\mu_{0} I_{\mathrm{sc}}}{2 \pi} \frac{\left(-y^{\prime}, x^{\prime}\right)}{x^{\prime 2}+y^{\prime 2}},
$$

with $I_{\mathrm{sc}}$ the superconducting current along the elementary wire centered at the source point $\left(x_{j}, y_{j}\right)$, and again $\left(x^{\prime}, y^{\prime}\right)$ standing for relative field-source coordinates. Based upon (7) and (8), Eq. (6) is specified by introducing tangential projections of the fields at each point of interest $G_{i j}=\overrightarrow{\mathscr{B}}\left(x_{i j}, y_{i j}\right)$. $\vec{\tau}\left(x_{i}, y_{i}\right)$. Thus, defined numerical model is ready to investigate the magnetic behavior of the heterostructure. To start with, we compare our numerical results to the analytical calculations in previous work. As figures of merit, we choose the values of the full penetration and cloaking fields obtained from Ref. 10 


$$
\begin{gathered}
\mathscr{H}_{\mathrm{fp}}=\frac{2 J_{c} R_{1}}{\pi}\left[1+\frac{\left(\mu_{r}-1\right)\left(2 \mu_{r}-1\right)\left(1-R_{1}^{2} / R_{2}^{2}\right)}{6 \mu_{r}}\right], \\
\mathscr{H}_{\text {cloak }}=\frac{8 J_{c} R_{1}}{3 \pi} \frac{\mu_{r}}{\mu_{r}^{2}-1} \frac{R_{1}^{2}}{R_{2}^{2}-R_{1}^{2}},
\end{gathered}
$$

with $\mu_{r}$ the relative permeability of the sheath and $R_{1}$ and $R_{2}$ the corresponding inner and outer radii.

Fig. 1 shows that the numerical results check very well against analytics when comparable. Dimensionless units ( $h \equiv \mathscr{H} / J_{c} R_{1}$ and $m \equiv \mathscr{M} / J_{c} R_{1}^{2}$ ) have been used. Concerning the determination of $\mathscr{H}_{\mathrm{fp}}$, we applied a differential criterion for dictating that the superconductor has reached saturation. Notice that Eq. (10) gives $\mathscr{H}_{\text {cloak }}$ provided that the superconductor is fully penetrated. For the case depicted, it can be shown ${ }^{10}$ that in such regime, cloaking may occur within the range $1<\mu_{r}<1.61$. Recall, however, that the equation may be safely used for $\mu_{r} \leqslant 2$.

Fig. 2 demonstrates the invisibility property for our system under partial and full penetration conditions. As indicated in Fig. 1, the cloaking condition is realized by the full magnetic moment of the system going to zero. In our long sample geometry, this is accompanied by the absence of magnetic moments of order higher than the dipole term. From the physical point of view, cloaking may be conveniently visualized by the magnetic contribution of the heterostructure. Fig. 3 reveals the property of the system "closing on itself" and thus leaving the applied field unperturbed in

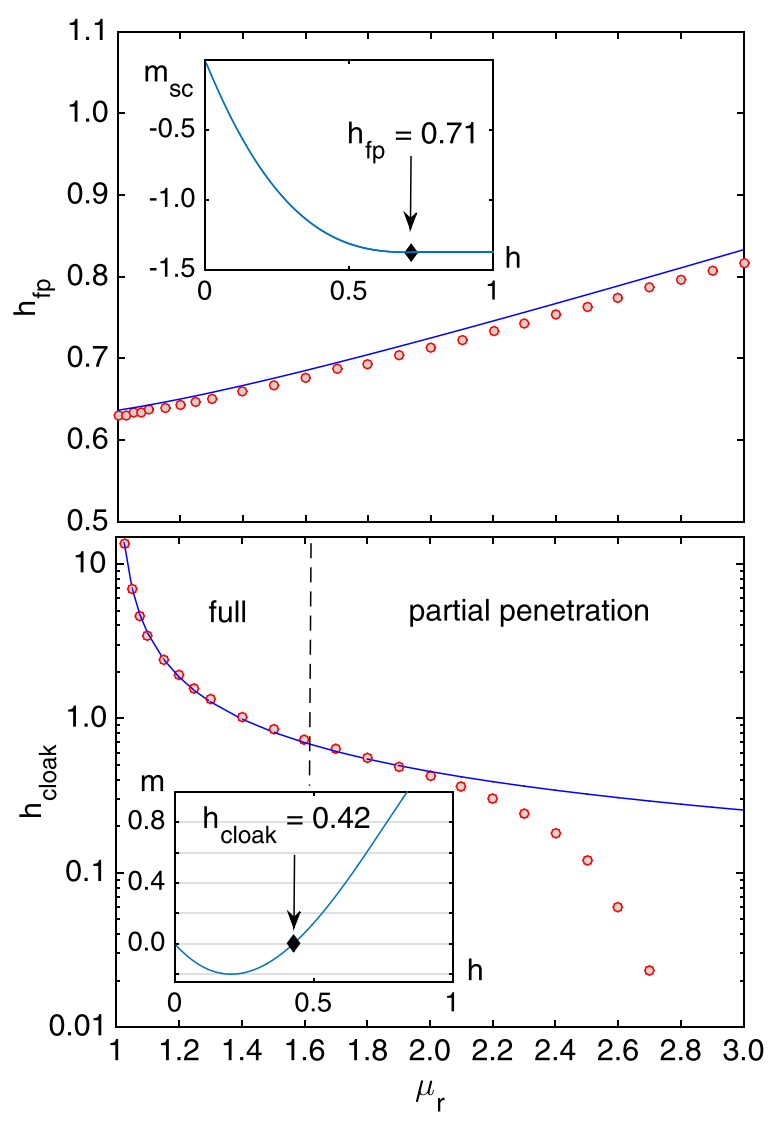

FIG. 1. Comparison of the numerical results (symbols) to the analytical expressions (lines) of the full penetration (upper panel) and cloaking (lower panel) fields for a paramagnetic sheath with $R_{2}=1.5 R_{1}$. Insets illustrate the procedure used to determine such fields for each value of $\mu_{r}$.
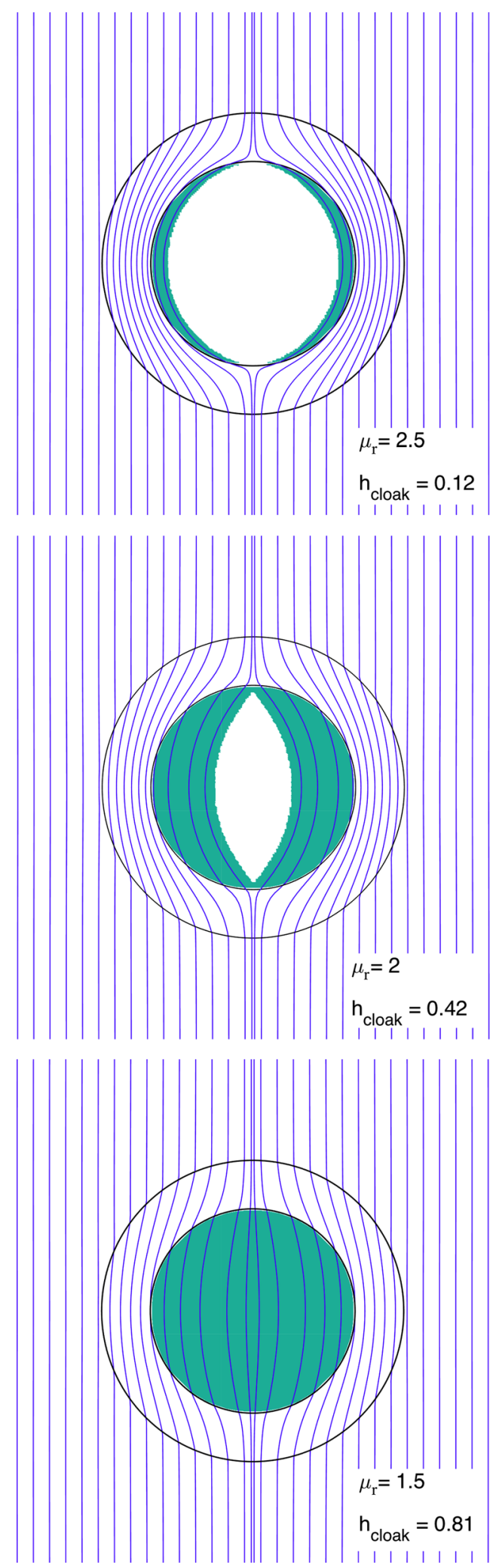

FIG. 2. Demonstration of the invisibility condition for different values of the magnetic permeability and always for $R_{2}=1.5 R_{1}$.

the surrounding space. Another aspect that has been considered here is the behavior of $\mathscr{H}_{\text {cloak }}$ as $\mu_{r}$ increases for a given geometry. According to Fig. 1, one could expect that $\mathscr{H}_{\text {cloak }}$ goes to zero at some finite value, say, $\mu_{r}^{*}$. Such value is the highest permeability for which magnetic cloaking may occur. This is clearly observed in Fig. 4 that displays the full magnetic moment for $R_{2} / R_{1}=1.5$ and a collection of values 

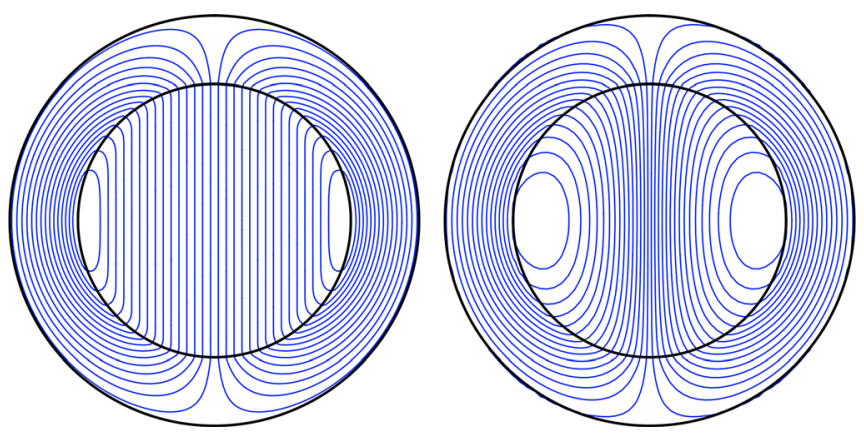

FIG. 3. Magnetic field lines contributed by the heterostructure itself in the invisibility condition. The plot shows the cases $\mu_{r}=2.5$ (left) and $\mu_{r}=1.5$ (right) in Fig. 2.

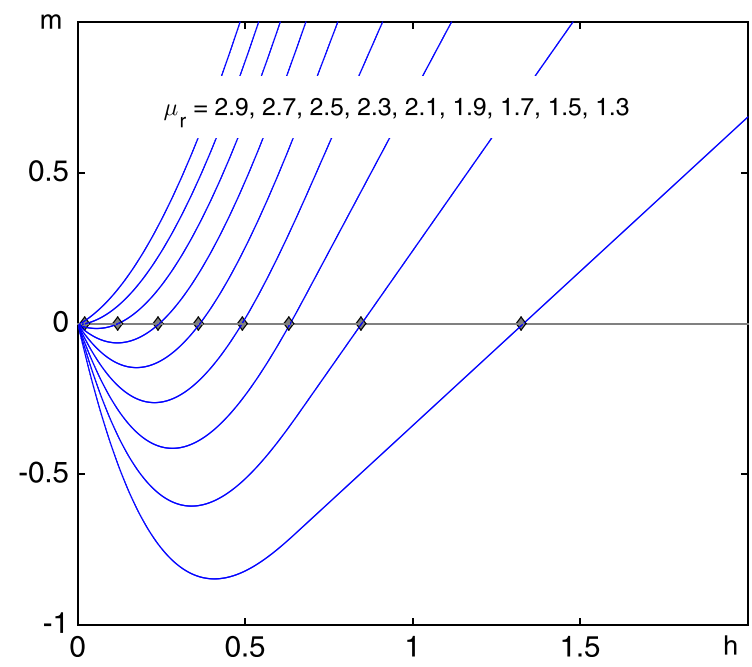

FIG. 4. Evolution of the full magnetic moment of the heterostructure in terms of the sheath permeability.

of $\mu_{r}$. One can notice that for low fields, $m$ is dominated by the superconductor's magnetization, whereas the paramagnetic behavior prevails for higher fields. As $\mu_{r}$ increases, the low field diamagnetic range is progressively reduced. Eventually, it disappears for a certain value $\mu_{r}^{*}$. This threshold is the real limit for the appearance of cloaking and always occurs with the superconductor within the partial penetration regime. When the penetration of magnetic flux in the superconductor is very small (low fields, as compared to
$\mathscr{H}_{\mathrm{fp}}$ ), like in upper panel of Fig. 2, cloaking is realized at a permeability value rather close to the estimation $\mu_{r}^{*}=\left(R_{2}^{2}+R_{1}^{2}\right) /\left(R_{2}^{2}-R_{1}^{2}\right)=2.6$ based on the perfect diamagnetism approximation. ${ }^{3}$ In fact, according to the resolution in Fig. 4, low field cloaking may occur in some point between $\mu_{r}^{*}=2.5$ and $\mu_{r}^{*}=2.7$.

Concluding, the variational critical state approach allowed investigation of magnetic invisibility of the cylindrical superconductor/paramagnet heterostructure in a wide range of magnetic field magnitudes including partially penetration regime. The results go far beyond the previous analysis based either on the perfect diamagnetism or on the full penetration assumptions and provide realistic magnitudes of the magnetic field and permeability required for the cloaking phenomenon.

Funding by Spanish MINECO and FEDER program (ENE2014-52105-R-9 project), DGA Grant No. T12, and partial support by the German DFG within the collaborative research center SFB 595 are gratefully acknowledged.

${ }^{1}$ I. F. Lyuksyutov and V. L. Pokrovsky, Adv. Phys. 54, 67 (2005); A. Y. Aladyshkin, A. V. Silhanek, W. Gillijns, and V. V. Moshchalkov, Supercond. Sci. Technol. 22, 053001 (2009).

${ }^{2}$ A. Sánchez, C. Navau, J. Prat-Camps, and D. X. Chen, New J. Phys. 13, 093034 (2011).

${ }^{3}$ F. Gömöry, M. Solovyov, J. Šouc, C. Navau, J. Prat-Camps, and A. Sánchez, Science 335, 1466 (2012).

${ }^{4}$ J. Šouc, M. Solovyov, F. Gömöry, J. Prat-Camps, C. Navau, and A. Sánchez, New J. Phys. 15, 053019 (2013).

${ }^{5}$ M. Solovyov, J. Šouc, J. Kováč, F. Gömöry, E. Mikulášová, M. Ušáková, and E. Ušák, IEEE Trans. Appl. Supercond. 26, 0500206 (2016).

${ }^{6}$ C. Navau, J. Prat-Camps, O. Romero-Isart, J. I. Cirac, and A. Sánchez, Phys. Rev. Lett. 112, 253901 (2014).

${ }^{7}$ P. B. Zhou, G. T. Ma, H. Liu, C. Yang, Z. N. Wang, and T. Y. Gong, J. Supercond. Novel Magn. 29, 1747 (2016).

${ }^{8}$ Y. A. Genenko, H. Rauh, and S. Kurdi, J. Appl. Phys. 117, 243909 (2015).

${ }^{9}$ S. V. Yampolskii and Y. A. Genenko, Appl. Phys. Lett. 104, 033501 (2014).

${ }^{10}$ S. V. Yampolskii and Y. A. Genenko, Appl. Phys. Lett. 104, 143504 (2014).

${ }^{11}$ C. P. Bean, Phys. Rev. Lett. 8, 250 (1962).

${ }^{12}$ J. D. Jackson, Classical Electrodynamics (Wiley, New York, 1975).

${ }^{13}$ A. Badía-Majós and C. López, Phys. Rev. Lett. 87, 127004 (2001); A. Badía-Majós, C. López, and H. S. Ruiz, Phys. Rev. B 80, 144509 (2009).

${ }^{14}$ Notice that, owing to the nature of our method, that uses superposition of elementary magnetic field contributions (wires and strips), continuity of the normal component of the full magnetic field is straightforwardly satisfied. 